\title{
May critical molecular cross-talk between indoleamine 2,3-dioxygenase (IDO) and arginase during human aging be targets for immunosenescence control?
}

Ismael Dale Cotrim Guerreiro da Silva ${ }^{1 *}$, Dirce Maria Lobo Marchioni ${ }^{2}$, Antonio Augusto Ferreira Carioca ${ }^{2,3}$, Valquiria Bueno ${ }^{4}$ and Gisele Wally Braga Colleoni ${ }^{*^{*}}$ (i)

\begin{abstract}
Background: This study aimed to identify novel plasma metabolic signatures with possible clinical relevance during the aging process. A biochemical quantitative phenotyping platform, based on targeted electrospray ionization tandem mass spectrometry technology, was used for the identification of any eventual perturbed biochemical pathway by the aging process in prospectively collected peripheral blood plasma from 166 individuals representing the population of São Paulo city, Brazil.

Results: Indoleamine 2,3-dioxygenase (IDO) activity (Kyn/Trp) was significantly elevated with age, and among metabolites most associated with elevations in IDO, one of the strongest correlations was with arginase (Orn/Arg), which could also facilitate the senescence process of the immune system. Hyperactivity of IDO was also found to correlate with increased blood concentrations of medium-chain acylcarnitines, suggesting that deficiencies in betaoxidation may also be involved in the immunosenescence process. Finally, our study provided evidence that the systemic methylation status was significantly increased and positively correlated to IDO activity.

Conclusions: In the present article, besides identifying elevated IDO activity exhibiting striking parallel association with the aging process, we additionally identified increased arginase activity as an underlying biochemical disturbance closely following elevations in IDO. Our findings support interventions to reduce IDO or arginase activities in an attempt to preserve the functionality of the immune system, including modulation of myeloidderived suppressor cells (MDSCs), T cells, macrophages, and dendritic cells' function, in old individuals/patients.
\end{abstract}

Keywords: IDO, Arginase, Aging, Senescence, Immune system

\footnotetext{
* Correspondence: ismael.dale2@gmail.com; gcolleoni@unifesp.br

'Departament of Gynecology, Escola Paulista de Medicina, Federal University of São Paulo, São Paulo, Brazil

${ }^{5}$ Department of Clinical and Experimental Oncology, Escola Paulista de Medicina, Federal University of São Paulo, São Paulo, Brazil

Full list of author information is available at the end of the article
}

(c) The Author(s). 2021 Open Access This article is licensed under a Creative Commons Attribution 4.0 International License, which permits use, sharing, adaptation, distribution and reproduction in any medium or format, as long as you give appropriate credit to the original author(s) and the source, provide a link to the Creative Commons licence, and indicate if changes were made. The images or other third party material in this article are included in the article's Creative Commons. licence, unless indicated otherwise in a credit line to the material. If material is not included in the article's Creative Commons licence and your intended use is not permitted by statutory regulation or exceeds the permitted use, you will need to obtain permission directly from the copyright holder. To view a copy of this licence, visit http://creativecommons.org/licenses/by/4.0/. The Creative Commons Public Domain Dedication waiver (http://creativecommons.org/publicdomain/zero/1.0/) applies to the data made available in this article, unless otherwise stated in a credit line to the data. 


\section{Introduction}

Immunosenescence is the name of a group of complex alterations in both innate and adaptive arms of the immune system associated with the aging process, leading to a progressive loss in the ability to respond to infections and poor immunity after vaccination [1], both associated with a higher mortality rate in old individuals. The rise in its recognition is pertinent and timely, given the increasing average age and the corresponding failure to increase healthy life expectancy [2].

A low pro-inflammatory phenotype - or inflammaging - in association with an adequate anti-inflammatory profile, could allow people to reach advanced age without disabilities. Thus, remodeling the immune responses seems important to reduce age-related degenerative diseases, both inflammatory and/or neoplastic, to achieve successful aging [3].

The diversity of cells, molecules, and pathways involved in the remodeling of the immune system and their ability to influence each other, including the individual variability of the immune response, turns difficult to identify interventions to improve or maintain the immune function in older adults [4]. In recent years, numerous studies on the mechanisms underlying agerelated immune decline became the basis for some interventions such as the reduction of the latent/lytic viral load, by vaccination and/or antiviral drugs, to diminish CMV-related immunosenescence [1, 4]. Supporting this theory, evidence suggests that infections and frailty repeatedly cross each other pathophysiological ways and accelerate the aging process in a vicious circle [5].

Although it is a recent concept and still much discussed, immunosenescence/inflammaging duo (representing two sides of the same phenomenon) may not be harmful but may represent that the most successful changes will guarantee healthy longevity. In addition, $\mathrm{cu}-$ mulative data suggest that, without their existence, human longevity would be greatly shortened [6-8].

Inflammaging and metaflammation largely share the same molecular mechanisms, in which metaflammation can be conceptualized as a specific situation of chronic inflammation caused by nutrient excess. The gut microbiota has a central role in both metaflammation and inflammaging due to its ability to release inflammatory products, contribute to circadian rhythms, and crosstalk with other organs and systems. Therefore, Franceschi et al. [6] argue that chronic diseases are not only the result of aging and inflammaging but accelerate the aging process. Also, they propose the use of metabolic biomarkers that are capable of assessing biological versus chronological age in metabolic diseases [6].

Hence, this study aimed to identify novel plasma biomarkers and metabolic signatures with possible relevance during the aging process. A biochemical quantitative phenotyping platform based on targeted electrospray ionization tandem mass spectrometry technology (ESI-MS/MS) was used to aid in the identification of any eventual perturbed biochemical pathway in peripheral blood plasma from our population-based participants.

\section{Material and methods \\ Participants}

The study population was selected from the "Health Survey of Sao Paulo (ISA-Capital)" a cross-sectional population-based survey to assess health conditions among a representative sample of individuals living in the City of Sao Paulo, South-eastern Brazil, between 2008 and 2009. A complex probabilistic sampling, by conglomerates, based on census tracts and households that had already drawn in the National Household Sample Survey 2005 (https://www.cps.fgv.br/cps/bd/curso/ Household-Surveys-Short-Description-site.pdf) was used. Participants were composed of 166 volunteers (female $n=79$, male $n=87$ ) at ages ranging from 20 to 89 years $($ mean $=50.7$, median $=51)$ with available, prospectively collected, samples during the accomplishment of the "Health Survey of Sao Paulo (ISA-Capital)". Due to its population-based characteristics, it was possible to group and analyze systemic metabolic variations of participants, distributed in sixteen progressive subgroups according to increasing ages ranging from 20 to 89 years (Fig. 1A). All plasma samples were collected on at least an 8-h fast in EDTA-containing tubes, centrifuged at $1000-\mathrm{x}$ g for $10 \mathrm{~min}$, transferred to a new tube, and stored immediately at $-80^{\circ} \mathrm{C}$ until analysis.

\section{Metabolomic analysis}

Plasma samples stored at $-80^{\circ} \mathrm{C}$ were kept on dry ice during transportation. Absolute quantification $(\mu \mathrm{M} / \mathrm{L})$ of peripheral blood metabolites was achieved by targeted quantitative profiling of 186 annotated metabolites by electrospray ionization (ESI) tandem mass spectrometry (MS/MS) in plasma samples, using SCIEX 5500 QTRA PO (SCIEX, Darmstadt, German), blinded to any phenotype information, on a centralized, independent, fee-forservice basis at the quantitative metabolomics platform from BIOCRATES Life Sciences AG, Innsbruck, Austria (https://biocrates.com/) $[9,10]$.

The experimental metabolomics measurement technique is described in detail by patent US 2007/0004044 (accessible online at http://www.freepatentsonline.com/2 0070004044.html). Briefly, a targeted profiling scheme was used to quantitatively screen for fully annotated metabolites using multiple reaction monitoring, neutral loss, and precursor ion scans. Quantification of metabolite concentrations and quality control assessment was performed with the MetIQ software package 

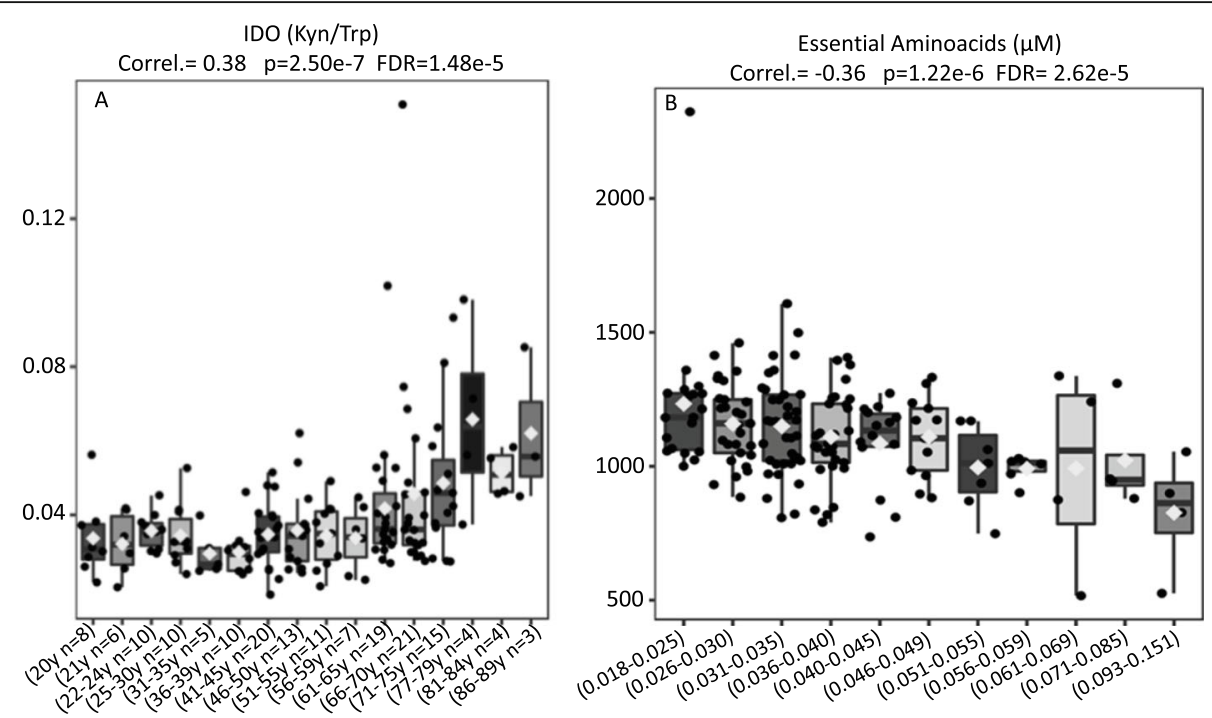

Fig. 1 A IDO activity (Kyn / Trp) (Y-Axis) is shown to be significantly elevated with age (X-Axis). B The sum of the essential amino acids (Y-Axis) shows an inverse correlation with IDO levels (X-Axis)

(BIOCRATES Life Sciences AG, Innsbruck, Austria) in conformance with 21CFR (Code of Federal Regulations) Part 11, which implies proof of reproducibility within a given error range. An xls file was then generated, which contained sample identification and 186 metabolite names and concentrations with the unit of $\mu \mathrm{mol} / \mathrm{L}$ of plasma (https://biocrates.com/) $[9,10]$.

In total, 186 annotated metabolites were quantified using the p180 kit (BIOCRATES Life Sciences AG, Innsbruck, Austria), being 40 acylcarnitines (AcylCs), 21 amino acids (AAs), 19 biogenic amines (BA), a sum of hexoses (Hex), 76 phosphatidylcholines (PCs), 14 lysophosphatidylcholines (LPCs) and 15 sphingomyelins (SMs). Glycerophospholipids were further differentiated with respect to the presence of ester (a) and ether (e) bonds in the glycerol moiety, where two letters denote that two glycerol positions are bound to a fatty acid residue $(\mathrm{aa}=$ diacyl, $\mathrm{ae}=\mathrm{acyl}$-alkyl), while a single letter indicates the presence of a single fatty acid residue $(\mathrm{a}=$ acyl or e = alkyl) (https://biocrates.com/) $[9,10]$.

For metabolomics data analysis, log-transformation was applied to all quantified metabolites to normalize the concentration distributions and uploaded into the web-based analytical pipelines MetaboAnalyst 3.0 (www. metaboanalyst.ca) and Receiver Operating Characteristic Curve Explorer \& Tester (ROCCET) available at http:// www.roccet.ca/ROCCET for the generation of uni and multivariate Receiver Operating Characteristic (ROC) curves obtained through Support Vector Machine (SVM), Partial Least Squares-Discriminant Analysis (PLS-DA) and Random Forests as well as Logistic Regression Models to calculate Odds Ratios of specific metabolites (http://www.roccet.ca/ROCCET) [9-13].
In addition to individual metabolite quantification, groups of metabolites related to specific functions were assembled as ratios based on the previous observation that the proportions between metabolite concentrations can strengthen the association signal and at the same time provide new information about possible metabolic pathways [14-16]. IDO activity was assessed through Kyn/Trp ratio and arginase activity by Orn/Arg ratio, with or without age correction.

\section{Results}

The indoloxigenase activity, as evaluated by the proportions between kynurenine to the essential amino acid tryptophan (Kyn/Trp), depicted the highest positive correlation with aging (Fig. 1A) (Correl. $=0.38, p=2.50 \mathrm{e}-7$, $\mathrm{FDR}=1.48 \mathrm{e}-5$ ), suggesting that the aging process seems to be driven by deficiencies in auxotrophic-related mechanisms, involving essential amino acids like tryptophan. To further confirm this possibility, we hypothesized that the concentration of essential amino acids should depict an inverse correlation pattern to increases in IDO activity.

In agreement with this hypothesis, the molar sum of the 9 essential amino acids (Val, Trp, Thr, Phe, Ile, Leu, Met, Lys, and His) exhibited an opposite and significant correlation with IDO activity (Correl. $=-0.36, p=1.22 \mathrm{e}$ $6, \mathrm{FDR}=2.62 \mathrm{e}-5)$, showing that nutritional aspects seem to matter in this group of individuals (Fig. 1B). Another important mechanism related to auxotrophy was identified in the present study: elevations in arginase activity, as evidenced by the proportions of ornithine to agenormalized arginine [Orn/(Arg/Age)], were significantly and positively correlated to IDO activity (Fig. 2A). 


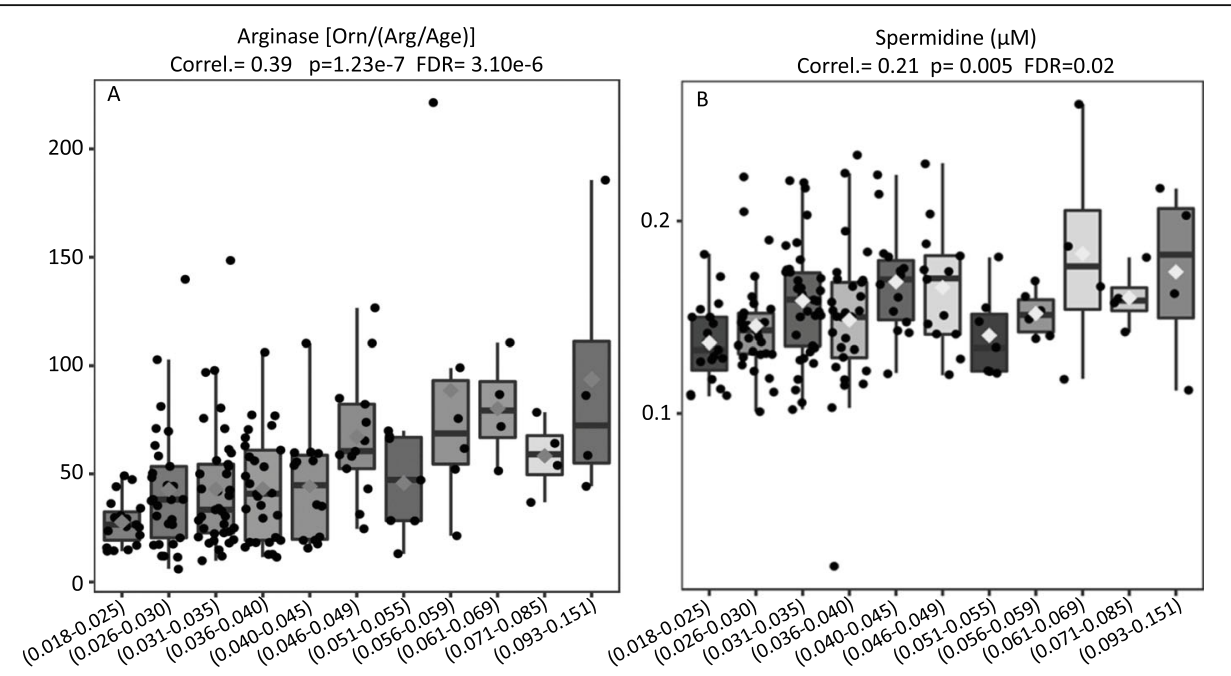

Fig. 2 A Elevations in arginase activity, evidenced by the proportions of ornithine to age-normalized arginine [Orn/(Arg/Age)] (Y-Axis), were significantly and positively correlated to IDO activity (X-Axis). B The molar levels of spermidine (Y-Axis) were significantly correlated to increases in IDO activity (X-Axis)

To further validate these results and knowing beforehand that the activation of arginase and IDO are capable to trigger spermidine production, we postulated that the levels of this biogenic amine should also be elevated. In total support of our hypothesis, the molar levels of spermidine were significantly correlated to increases in IDO activity (Fig. 2B).

Hyperactivity of indoloxigenase was also found to correlate with increased blood concentrations of mediumchain acylcarnitines, such as decadienylcarnitine (C10:2); this finding suggests that deficiencies in beta-oxidation may also be involved in the immunosenescence process
(Fig. 3A) $($ Correl. $=0.53, p=1.25 \mathrm{e}-13, \mathrm{FDR}=4.23 \mathrm{e}-12)$. To confirm this possibility, we assembled the ratio between the total amounts of acylcarnitines (AcylC) to the levels of free carnitine (CO). In complete alignment with our hypothesis, the increased values generated by the ratio $(\mathrm{AcylC} / \mathrm{CO})$ revealed that blood acylcarnitines (AcylC) concentrations are disproportionally elevated concerning free carnitine (CO). As such, these biochemical deviations are highly suggestive that beta-oxidation deficiencies are, indeed, associated to increased values in IDO activity (Fig. 3B) (Correl. $=0.46, p=2.40 \mathrm{e}-10$, FDR $=3.80 \mathrm{e}-9$ ).

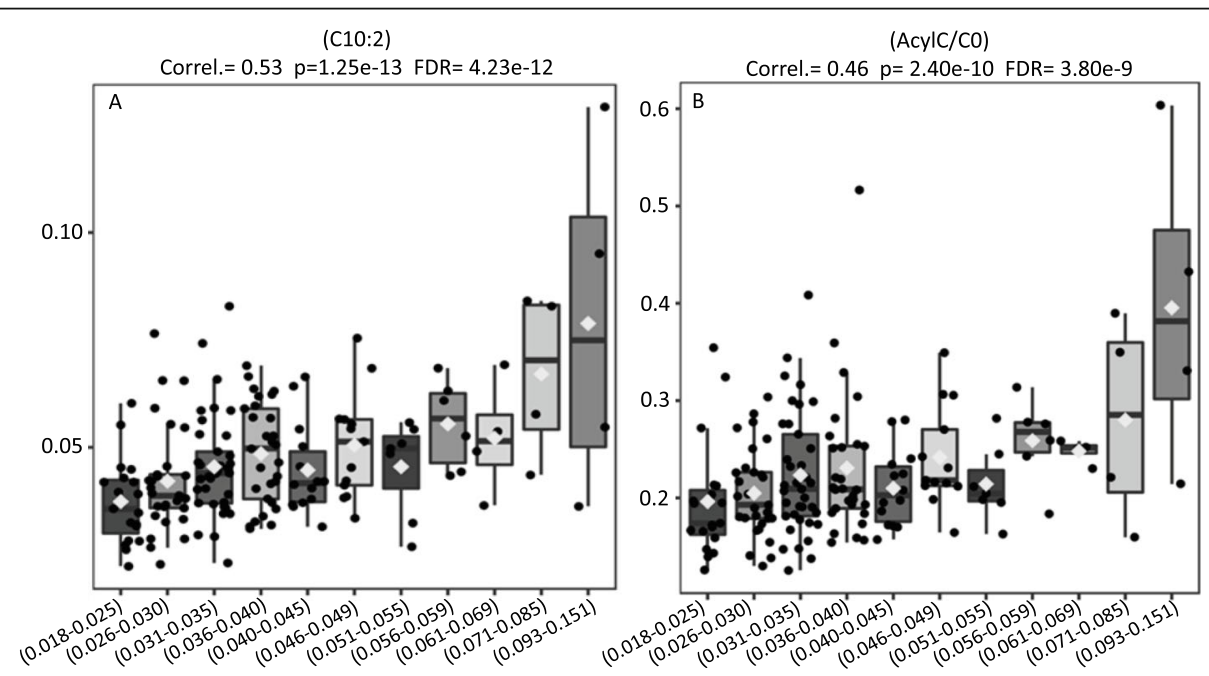

Fig. 3 A Elevations in the levels of medium-chain acylcarnitines such as C10: 2 (Y-Axis) are associated with elevations in the IDO (X-Axis). B Ratio between the total amounts of acylcarnitines (AcylC) to the levels of free carnitine (CO (AcylC/CO) revealed that blood acylcarnitines (AcylC) concentrations are disproportionally elevated concerning free carnitine (CO), suggesting that beta-oxidation deficiencies are associated with increased values in IDO activity (X-axis) 
Finally, our study provided evidence that the systemic methylation status was found significantly increased and positively correlated to IDO activity (Correl. $=0.63, p=$ $3.49 \mathrm{e}-20, \mathrm{FDR}=3.56 \mathrm{e}-18)$ as evaluated by the proportions between the molar concentrations of Total Dimethylated Arginine (Total DMA) to its unmodified arginine values (corrected for age) (Fig. 4).

\section{Discussion}

Some amino acids catabolic pathways, as well as normal mitochondrial function, have become critical checkpoints in immunity [17-19]. The necessity for an external source of a nutrient is called auxotrophy. Amino acid auxotrophy is known nowadays to constitute a fundamental immunoregulatory control node that modulates immune responses mostly by tempering $\mathrm{T}$ cell activity through a diversity of mechanisms including amino acid starvation [18]. From the amino acids perspective, myeloid-derived suppressor cells (MDSCs) mediate their suppressive activity by promoting the production of arginase and IDO, leading to deprivation of amino acids required for immune cell activation and proliferation [17]. Many amino acids have been found to play important roles as immune regulatory players, including ornithine (Orn), tryptophan (Trp), kynurenine (Kyn), and arginine (Arg) [18]. Therefore, the associated immunoregulatory effects rely on the depletion of specific amino acids in the microenvironment and/or generation of biologically active metabolites [20].

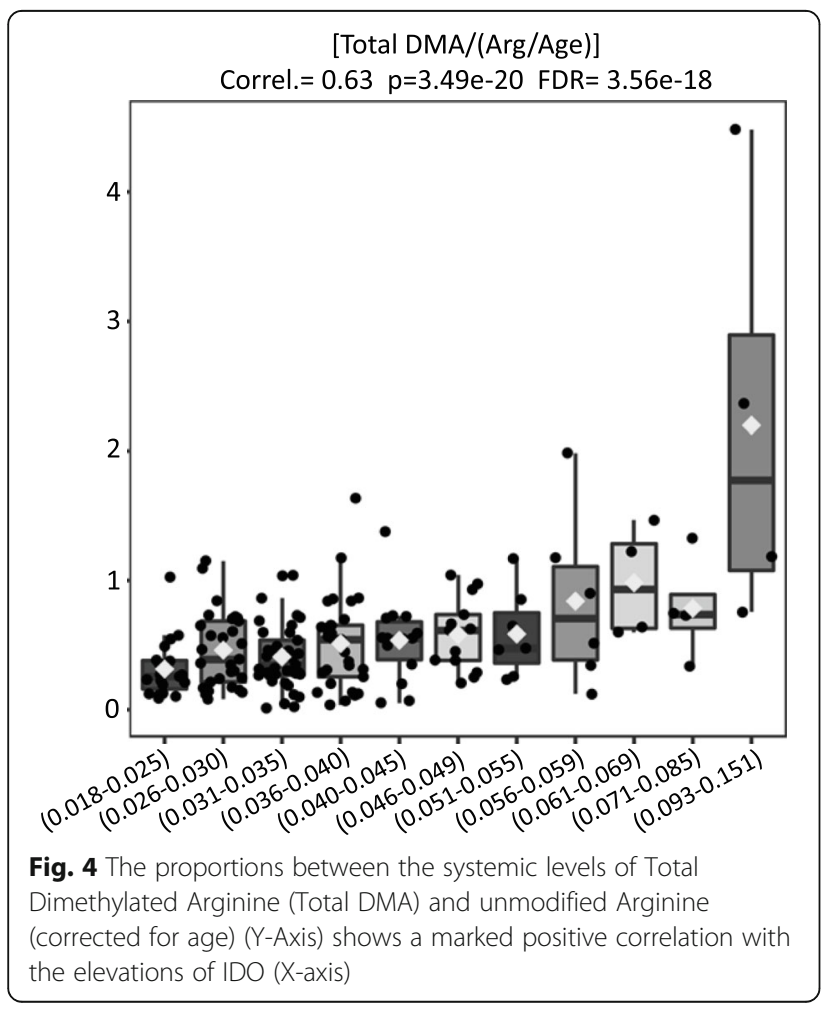

In the present article, besides identifying elevated IDO (Kyn/Trp) activity, exhibiting striking parallel association to the aging processes, we additionally identified increased arginase (Orn/Arg) activity as an underlying biochemical disturbance closely following elevations in IDO. Indeed, the sequential activation of arginase and IDO, promotes a potent immunoregulatory phenotype in which spermidine, a polyamine produced downstream of the arginase-dependent pathway, is capable of triggering IDO phosphorylation and signaling, representing the critical molecular interconnection between the two enzymes [20]. Of note, enzymes that hydrolyze Ltryptophan and that degrade L-arginine are substantially increased in cancer [21], highlighting the potential of these two pathways/enzyme inhibitors in improving immune response in cancer and other immunosuppressive conditions, and contributing to a healthy aging.

The enzyme IDO is responsible for the rate-limiting step of tryptophan catabolism. Metabolites generated from tryptophan catabolism are immunoregulatory molecules, collectively called kynurenines, playing important roles in modulating immune cell function [20]. The role of tryptophan metabolism in immune function has recently become an area of intense study as it relates to the propensity of tumor cells to evade immune cell responses. Enforced expression of IDO in tumor cells impaired the antitumor immune responses by $\mathrm{T}$ cells, but this could be overcome by pharmacological inhibition of IDO with 1-methyltryptophan [22, 23]. Therefore, amino acid metabolism in immune cells could yield important new insights into immune cell function [24]. Reinforcing that IDO activity might be a mechanism involved in the decline of $\mathrm{T}$ cell responses in immunosenescence, Pertovaara et al. [25] measured Kyn/Trp, reflecting IDO activity, in 284 nonagenarians and 309 blood donor controls. IDO activity was significantly higher in nonagenarians compared with young controls and predicted subsequent mortality in the group of old individuals.

From the standpoint of energy production and mitochondrial function, recent studies have shown that different immune cell subtypes use distinct metabolic programs to perform their functions [19]. Indeed, effector $\mathrm{T}$ cells prioritize aerobic glycolysis during anabolic metabolism to balance the synthesis of macromolecules and the generation of energy to support it. Conversely, memory $\mathrm{T}$ cells, as well as regulatory $\mathrm{T}$ cells (Treg), prioritize fatty acid oxidation (FAO also called beta-oxidation) to support the energy demand for survival and function [26]. Of note, in the present article, we identified disturbances in beta-oxidation as another underlying condition depicting significant correlations to IDO activity. Indeed, the elevated proportions between the sum of esterified carnitines (AcylC) to free carnitine (C0) 
significantly parallels the increasing levels in IDO activity (Fig. 3B).

L-Arginine is a nonessential amino acid metabolized by arginase 1 (Arg1), arginase 2 , and inducible nitric oxide (NO) synthase. Arg1 and arginase 2 hydrolyze LArginine into urea and L-ornithine, the latter being the main substrate for the production of polyamines that are required for cell cycle progression. Therefore, the accumulation of ornithine and polyamines facilitates cancer cell proliferation. Besides its fundamental role in the hepatic urea cycle, arginase is also expressed in the immune system of mice and men [27].

L-Arginine is also metabolized by inducible NO synthase to citrulline and $\mathrm{NO}$, a highly reactive compound important in vascular homeostasis, and as part of the cytotoxic mechanism of macrophages [28]. Therefore, arginine metabolism has been shown to play a key role in the inflammatory function of macrophages [29]. Macrophages use arginine in two distinct metabolic pathways, the NO synthesis, and the arginase pathways. The pathway used for arginine metabolism in macrophages has profound effects on the immune function of the cell [24]. Macrophage flux of arginine into the NO synthesis pathway is associated with an inflammatory M1 phenotype. In contrast, arginine flux through the arginase pathway is associated with a more tolerant immune response [24]. Difluoromethylornithine, an inhibitor of ornithine decarboxylase has demonstrated promising antitumor effects in mouse models of prostate cancer [30]. Therefore, a reduction in L-arginine may be related to immunosuppression associated with cancer and aging and could be supplemented in an attempt to reverse this deviation [27]. Arginase is also constitutively expressed by human polymorphonuclear granulocytes (PMN) and severely impairs key functions of primary human NK cells as well as IL-2-activated NK cells. In the absence of arginine, NK cell proliferation and IL-12/IL-18-induced secretion of IFN-gamma are severely diminished [31].

Therefore, Arg1 and IDO1 are immunosuppressive enzymes known to operate in distinct immune cells. Mondanelli et al. [32] demonstrate that Arg1 and IDO1 cooperate in conferring long-term, immunosuppressive effects on dendritic cells and exert proapoptotic and antiproliferative effects on T-cells, either by a shared, welldescribed molecular pathway, but also by other separate mechanisms [33].

Arginine methylation is known to play a major role in gene regulation because of the ability of the predominant methyltransferase (PRMTs) to deposit key activating (histone H4R3me2a, H3R2me2s, H3R17me2a, H3R26me2a) or repressive (H3R2me2a, H3R8me2a, H3R8me2s, H4R3me2s) histone marks. In addition, there are many substrates that are non-histones involved in biological processes including transcription, cell signaling, mRNA translation, DNA damage signaling, receptor trafficking, protein stability, and pre-mRNA splicing [34, 35]. Therefore, arginine methylation has emerged as a key regulator of signal transduction with an important role in $\mathrm{T}$ lymphocyte activation. The PRMT-1 is highly expressed in $\mathrm{T}$ helper cells, and ligation of the $\mathrm{T}$ cell antigen and costimulatory receptors induces arginine methylation on several cytoplasmic proteins. Global inhibition of methyltransferases can result in signaling defects in CD4+ T cells and profound immunosuppression. Here we suggest that manipulating arginine methylation could be a feasible strategy to modulate $\mathrm{T}$ lymphocyte function, presenting a novel approach towards immunotherapy and the treatment of $\mathrm{T}$ cell-mediated disorders such as autoimmune disease and transplant rejection [36].

Besides IDO1 and Arg 1, other amino acid degrading enzymes such as tryptophan 2,3-dioxygenase and arginase 2, has relevance in the regulation of tumor-induced immune tolerance, including the induction of an immunosuppressive tumor microenvironment [23]. IDO1mediated tryptophan degradation can promote Treg cell differentiation and activation, which, in turn, leads to the expansion and intratumoral recruitment of Argcompetent immunosuppressive myeloid populations like myeloid-derived suppressor cells (MDSCs). Preclinical data suggest that IDO1 inhibition by INCB024360 will increase $\mathrm{T}$ cell proliferation, decrease Treg cells and MDSCs activity. In Phase II clinical trial in myelodysplastic syndrome patients, although the mean kynurenine/tryptophan ratio decreased $42 \%$ at cycle 2 , the alterations in MDSCs and T effector cells were not significant and $80 \%$ of patients achieved only stable disease as the best response [37].

MDSCs significantly increase with aging and are the enhancers of other immunosuppressive cells, such as Treg and regulatory B cells (Bregs). Therefore, one could think that MDSCs remodel the immune system, preventing excessive inflammation with aging and inducing immunosenescence [38].

MDSCs cause suppression of immune cells via several mechanisms, notably through reactive oxygen species (ROS) generated by g-MDSCs, NO generated by $\mathrm{m}$ MDSCs, production of arginase, and also by secretion of cytokines such as IL-10 and TGF- $\beta$. Arginine is used metabolically by MDSC and thus by removing this substrate from the microenvironment that is also used by $\mathrm{T}$ cells, MDSC prevents its proliferation [39]. MDSCs can then further inhibit antitumor effector T-cell responses through arginase-, iNOS-, PD-L1- and inhibitory cytokine-dependent mechanisms [23]. Thus, the consumption of L-arginine by Arg 1 represents a wellknown immunoregulatory mechanism exploited by M2 macrophages and MDSCs [20]. 


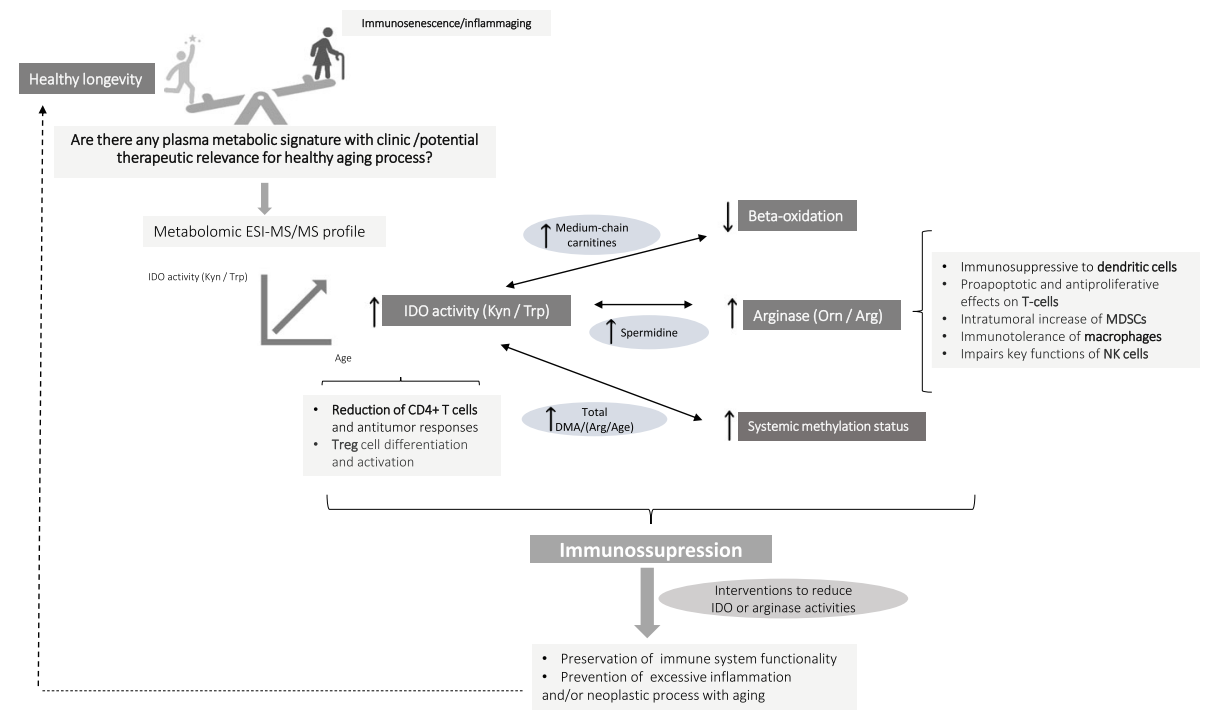

Fig. 5 Study overview and literature revision, as described in the discussion section

Observed age-associated dysfunction of macrophages is the result of their functional adaptation to the ageassociated changes in tissue environments. The macrophages appear to maintain functional plasticity during this dysregulation, making them a prime target of cytokine therapy that could enhance both innate and adaptive immune systems [40]. In cancer, however, both ARG1 and IDO1 are overexpressed, in MDSCs and DCs, and contribute to the impairment of the host anti-tumor immunity [20]. Therefore, is ARG1 inhibition a good therapeutic strategy? The use of arginase inhibitors such as nor-N-hydroxy-L-arginine abrogated the arresting effects of arginase on T-cell proliferation and allowed lymphocyte-dependent tumor reduction [28]. As MDSCs are also able to polarize macrophages toward the M2 phenotype, arginase inhibitors could have a double effect in favor of $\mathrm{T}$ cells proliferation and preventing the expansion of immunosuppressive tumor-associated macrophages (TAMs) [41]. Further investigations on arginine metabolism in the aging process, neoplasia, and also autoimmune disorders, and its possible relationship with IDO1 is a new pathway to be explored, with possible therapeutic purposes (Fig. 5).

One limitation of this study is to hypothesize that metabolomic findings related to aging are also related to immunosenescence. However, the correlations are entirely theoretical and based on literature data. Future studies with immune subpopulations could reinforce the present hypothesis.

In conclusion, in the present article, besides identifying elevated IDO activity exhibiting striking parallel association with the aging process, we additionally identified increased arginase activity as an underlying biochemical disturbance closely following elevations in IDO. Our findings support interventions to reduce IDO or arginase activities in an attempt to preserve the functionality of the immune system, including modulation of MDSCs, T cells, macrophages, and dendritic cells' function, in old individuals/patients.

\section{Abbreviations \\ AAs: Amino acids; AcylC: Acylcarnitines; Arg1: Arginase 1; BA: Biogenic amines; Breg: Regulatory B cells; C10:2: Decadienylcarnitine; CO: Free carnitine; EDTA: Ethylenediaminetetraacetic acid; ESI-MS: Electrospray ionization tandem mass spectrometry; FAO: Fatty acid oxidation or $\beta$ - oxidation; FDR: False discovery rates; Hex: Sum of hexoses; IDO: Indoleamine 2,3-dioxygenase; ISA-Capital: Health Survey of Sao Paulo; Kyn/Trp: Kynurenine to tryptophan ratio; LPCs: Lyso-phosphatidylcholines; MCCV: Monte-Carlo Cross Validation; MDSCs: Myeloid-derived suppressor cells; MS: Mass spectrometry; NO: Nitric oxide; Orn/Arg: Ornithine to arginine ratio; PCs: Phosphatidylcholines; PLS-DA: Partial Least Squares-Discriminant Ana- Iysis; PMN: Polymorphonuclear granulocytes; PRMTs: Predominant methyl transferase; ROC: Receiver Operating Characteristic; ROS: Reactive oxygen species; SMs: Sphingomyelins; SVM: Support Vector Machine; TAMs: Tumor associated macrophages; Treg: Regulatory $T$ cells}

\section{Acknowledgments}

The authors thank Sao Paulo Population Public Health Research Project (ISA 2008), for collaboration with healthy control group composition.

\section{Authors' contributions}

I.D.C.G.S., V.B., and G.W.B.C. were responsible for work design, acquisition, analyses, and interpretation of data, and wrote the manuscript; D.M.L.M. and A.A.F.C. were responsible for data acquisition. The authors read and approved the final manuscript.

\section{Funding}

This work was supported by Fundaçao de Amparo à Pesquisa do Estado de São Paulo (FAPESP), Brazil (2017/21801-2) and Coordenação de Aperfeiçoamento de Pessoal de Nível Superior, Finance code 001 - Brasil (CAPES).

\section{Availability of data and materials}

Our raw data was deposit in Metabolights (www.ebi.ac.uk/metabolights/ MTBLS2260). 


\section{Declarations}

\section{Ethics approval and consent to participate}

This study was approved by the Research Ethics Committee of the Universidade Federal de São Paulo - UNIFESP (\#1123/2018). All healthy controls provided written informed consent before plasma processing. We confirm that all the experimental protocols were under the guidelines of the national/institutional or Declaration of Helsinki.

\section{Consent for publication}

All authors agree to publish the present data.

\section{Competing interests}

We declare no conflicts to disclose.

\section{Author details}

'Departament of Gynecology, Escola Paulista de Medicina, Federal University of São Paulo, São Paulo, Brazil. ${ }^{2}$ Nutrition Department, School of Public Health, University of São Paulo School of Medicine (FMUSP), São Paulo, Brazil. ${ }^{3}$ Nutrition Department, Universidade de Fortaleza (UNIFOR), Fortaleza, Brazil. ${ }^{4}$ Department of Microbiology, Immunology and Parasitology, Escola Paulista de Medicina, Federal University of São Paulo, São Paulo, Brazil. ${ }^{5}$ Department of Clinical and Experimental Oncology, Escola Paulista de Medicina, Federal University of São Paulo, São Paulo, Brazil.

\section{Received: 10 May 2021 Accepted: 2 August 2021}

\section{Published online: 13 August 2021}

\section{References}

1. Crooke SN, Ovsyannikova IG, Poland GA, Kennedy RB. Immunosenescence and human vaccine immune responses. Immun Ageing. 2019. https://doi. org/10.1186/s12979-019-0164-9.

2. Aw D, Silva $A B$, Palmer DB. Immunosenescence: emerging challenges for an ageing population. Immunology. 2007. https://doi.org/10.1111/j.1365-2567.2 007.02555.x

3. Ventura MT, Casciaro M, Gangemi S, Buquicchio R. Immunosenescence in aging: between immune cells depletion and cytokines up-regulation. Clin Mol Allergy. 2017. https://doi.org/10.1186/s12948-017-0077-0.

4. Aiello A, Farzaneh F, Candore G, et al. Immunosenescence and its hallmarks: how to oppose aging strategically? A review of potential options for therapeutic intervention. Front Immunol. 2019. https://doi.org/10.3389/ fimmu.2019.02247

5. Vetrano DL, Triolo F, Maggi S, Malley R, Jackson TA, Poscia A, et al. Fostering healthy aging: the interdependency of infections, immunity and frailty. Ageing Res Rev. 2021. https://doi.org/10.1016/j.arr.2021.101351.

6. Franceschi C, Garagnani P, Parini P, Giuliani C, Santoro A. Inflammaging: a new immune-metabolic viewpoint for age-related diseases. Nat Rev Endocrinol. 2018. https://doi.org/10.1038/s41574-018-0059-4.

7. Fulop T, Larbi A, Dupuis $G$, et al. Immunosenescence and Inflamm-aging as two sides of the same coin: friends or foes? Front Immunol. 2018. https:// doi.org/10.3389/fimmu.2017.01960.

8. Pawelec G. Age and immunity: what is "immunosenescence"? Exp Gerontol. 2018. https://doi.org/10.1016/j.exger.2017.10.024

9. da Silva I, et al. Inborn-like errors of metabolism are determinants of breast cancer risk, clinical response and survival: a study of human biochemical individuality. Oncotarget. 2018. https://doi.org/10.18632/oncotarget.25839.

10. da Silva IDCG, Levatti EVC, Pedroso AP, et al. Biochemical Phenotyping of multiple myeloma patients at diagnosis reveals a disorder of mitochondrial complexes I and II and a Hartnup-like disturbance as underlying conditions, also influencing different stages of the disease. Sci Rep. 2020. https://doi. org/10.1038/s41598-020-75862-4.

11. Altmaier $\mathrm{E}$, et al. Bioinformatics analysis of targeted metabolomics-uncovering old and new tales of diabetic mice under medication. Endocrinology. 2008. https://doi.org/10.1210/en.2007-1747.

12. Bartel J, et al. The human blood Metabolome-Transcriptome Interface. PLoS Genet. 2015. https://doi.org/10.1371/journal.pgen.1005274.

13. Gieger C, Geistlinger L, Altmaier $\mathrm{E}$, et al. Genetics meets metabolomics: a genome-wide association study of metabolite profiles in human serum. PLoS Genet. 2008;4(11):e1000282. https://doi.org/10.1371/journal. pgen.1000282.
14. Illig T, et al. A genome-wide perspective of genetic variation in human metabolism. Nat Genet. 2010. https://doi.org/10.1038/ng.507.

15. Suhre $K$, Shin SY, Petersen AK, et al. Human metabolic individuality in biomedical and pharmaceutical research. Nature. 2011. https://doi.org/10.1 038/nature10354.

16. Chong J, et al. MetaboAnalyst 4.0 for comprehensive and integrative metabolomics data analysis. Curr Protoc Bioinformatics. 2019. https://doi. org/10.1002/cpbi.86.

17. Gabrilovich DI, Nagaraj S. Myeloid-derived suppressor cells as regulators of the immune system. Nat Rev Immunol. 2009. https://doi.org/10.1038/nri2 506.

18. Murray PJ. Amino acid Auxotrophy as immunological control nodes. Nat Immunol. 2016:17:132

19. Breda CNS, Davanzo GG, Basso PJ, Saraiva Câmara NO, Moraes-Vieira PMM Mitochondria as central hub of the immune system. Redox Biol. 2019;26: 101255

20. Mondanelli G, lacono A, Allegrucci M, Puccetti P, Grohmann U. Immunoregulatory interplay between arginine and tryptophan metabolism in health and disease. Front Immunol. 2019. https://doi.org/10.3389/fimmu.2 019.01565.

21. Grzywa TM, Sosnowska A, Matryba $P$, et al. Myeloid cell-derived Arginase in cancer immune response. Front Immunol. 2020. https://doi.org/10.3389/ fimmu.2020.00938.

22. Holmgaard RB, Zamarin D, Munn DH, Wolchok JD, Allison JP. Indoleamine 2,3-dioxygenase is a critical resistance mechanism in antitumor T cell immunotherapy targeting CTLA-4. J Exp Med. 2013. https://doi.org/10.1084/ jem.20130066

23. Timosenko E, Hadjinicolaou AV, Cerundolo V. Modulation of cancer-specific immune responses by amino acid degrading enzymes. Immunotherapy. 2017. https://doi.org/10.2217/imt-2016-0118

24. O'Neill LA, Kishton RJ, Rathmell J. A guide to immunometabolism for immunologists. Nat Rev Immunol. 2016. https://doi.org/10.1038/nri.2016.70.

25. Pertovaara $M$, Raitala $A$, Lehtimäki $T$, et al. Indoleamine 2,3-dioxygenase activity in nonagenarians is markedly increased and predicts mortality. Mech Ageing Dev. 2006. https://doi.org/10.1016/j.mad.2006.01.020.

26. Kouidhi S, Elgaaied AB, Chouaib S. Impact of metabolism on T-cell differentiation and function and cross talk with tumor microenvironment. Front Immunol. 2017. https://doi.org/10.3389/fimmu.2017.00270.

27. Munder M. Arginase: an emerging key player in the mammalian immune system. Br J Pharmacol. 2009. https://doi.org/10.1111/j.1476-5381.2009.002 91.x.

28. Rodriguez PC, Quiceno DG, Zabaleta J, et al. Arginase I production in the tumor microenvironment by mature myeloid cells inhibits T-cell receptor expression and antigen-specific T-cell responses. Cancer Res. 2004;64:5839.

29. Rath M, Müller I, Kropf P, Closs El, Munder M. Metabolism via Arginase or nitric oxide synthase: two competing arginine pathways in macrophages. Front Immunol. 2014. https://doi.org/10.3389/fimmu.2014.00532.

30. Gupta S, Ahmad N, Marengo SR, Maclennan GT, Greenberg NM, Mukhtar H. Chemoprevention of prostate carcinogenesis by alphadifluoromethylornithine in TRAMP mice. Cancer Res. 2000;60:5125.

31. Oberlies J, Watzl C, Giese T, et al. Regulation of NK cell function by human granulocyte arginase. J Immunol. 2009. https://doi.org/10.4049/jimmunol. 0803523.

32. Mondanelli G, Bianchi R, Pallotta MT, et al. A relay pathway between arginine and tryptophan metabolism confers immunosuppressive properties on dendritic cells. Immunity. 2017. https://doi.org/10.1016/j.immuni.2017.01. 005.

33. Eleftheriadis T, Yiannaki E, Antoniadi G, et al. Plasma indoleamine 2,3dioxygenase and arginase type I may contribute to decreased blood T-cell count in hemodialysis patients. Ren Fail. 2012. https://doi.org/10.3109/ 0886022X.2012.713297.

34. Auclair $Y$, Richard $S$. The role of arginine methylation in the DNA damage response. DNA Repair (Amst). 12(7):459-65. https://doi.org/10.1016/j.dna rep.2013.04.006 Epub 2013 May 17. PMID: 23684798 (2013).

35. Yang Y, Lu Y, Espejo A, Wu J, Xu W, Liang S, et al. TDRD3 is an effector molecule for arginine-methylated histone marks. Mol Cell. 2010. https://doi. org/10.1016/j.molcel.2010.11.024.

36. Parry RV, Ward SG. Protein arginine methylation: a new handle on T lymphocytes? Trends Immunol. 2010;31:164

37. Komrokji RS, Wei S, Mailloux AW, et al. A phase II study to determine the safety and efficacy of the Oral inhibitor of Indoleamine 2,3-Dioxygenase 
(IDO) enzyme INCB024360 in patients with Myelodysplastic syndromes. Clin Lymphoma Myeloma Leuk. 2019. https://doi.org/10.1016/j.clml.2018.12.005.

38. Salminen A, Kaarniranta K, Kauppinen A. Immunosenescence: the potential role of myeloid-derived suppressor cells (MDSC) in age-related immune deficiency. Cell Mol Life Sci. 2019. https://doi.org/10.1007/s00018-019-03048-x.

39. Bueno V, Sant'Anna OA, Lord JM. Ageing and myeloid-derived suppressor cells: possible involvement in immunosenescence and age-related disease. Age (Dordr). 2014. https://doi.org/10.1007/s11357-014-9729-x.

40. Stout RD, Suttles J. Immunosenescence and macrophage functional plasticity: dysregulation of macrophage function by age-associated microenvironmental changes. Immunol Rev. 2005. https://doi.org/10.1111/j. 0105-2896.2005.00260.X.

41. Mussai F, De Santo C, Abu-Dayyeh I, et al. Acute myeloid leukemia creates an arginase-dependent immunosuppressive microenvironment. Blood. 2013; $122 \cdot 749$.

\section{Publisher's Note}

Springer Nature remains neutral with regard to jurisdictional claims in published maps and institutional affiliations.

Ready to submit your research? Choose BMC and benefit from:

- fast, convenient online submission

- thorough peer review by experienced researchers in your field

- rapid publication on acceptance

- support for research data, including large and complex data types

- gold Open Access which fosters wider collaboration and increased citations

- maximum visibility for your research: over $100 \mathrm{M}$ website views per year

At BMC, research is always in progress.

Learn more biomedcentral.com/submissions 